\title{
The energy spectrum for stochastic eddies with gamma distribution
}

Rukiye KaraMine Caglar

Citation: AIP Conference Proceedings 1479, 213 (2012); doi: 10.1063/1.4756100

View online: http://dx.doi.org/10.1063/1.4756100

View Table of Contents: http://aip.scitation.org/toc/apc/1479/1

Published by the American Institute of Physics 


\title{
The Energy Spectrum for Stochastic Eddies with Gamma Distribution
}

\author{
Rukiye Kara* and Mine Caglar ${ }^{\dagger}$ \\ ${ }^{*}$ Mimar Sinan Fine Arts University, Department of Mathematics, Istanbul, TURKEY \\ ${ }^{\dagger}$ Koc University, Department of Mathematics, Istanbul, TURKEY
}

\begin{abstract}
Lundgren (1982) showed that strained spiral vortex model for turbulent fine structure has exponential Kolmogorov energy spectrum form. Caglar (2007) has generalized Cinlar velocity field which defined a similar structure with Lundgren vortex and computed the energy spectrum. In this study, we investigate the energy spectrum of the stochastic velocity field using Gamma distribution for small scale eddies.
\end{abstract}

Keywords: Stochastic flows; Kolmogorov spectrum; homogeneous turbulence.

PACS: 76F05,60E99

\section{INTRODUCTION}

The class of velocity fields introduced by Cinlar $[1,2]$ is motivated by small to medium scale eddies. They can generate a wide range of flows as well as the features of stationary and homogeneous turbulence $[3,4,5]$.

In this section, we review flows generated by Cinlar velocity fields [1-5]. Let $v$ be a deterministic velocity field on $\mathbb{R}^{2}$ called the basic eddy, and let $Q=\mathbb{R}^{2} \times \mathbb{R} \times(0, \infty)$ be an index set. Eddies of different sizes and amplitudes for $q \varepsilon Q, x \varepsilon \mathbb{R}^{2}$ are obtained by

$$
v_{q}(x)=a v\left(\frac{x-z}{b}\right), \quad q=(z, a, b)
$$

Let $N$ be a Poisson random measure on the Borel sets of $\mathbb{R} \times Q$ with mean measure

$$
\mu(d t, d q) \equiv \mu(d t, d z, d a, d b)=\lambda d t d z \alpha(d a) \beta(d b)
$$

where $\lambda$ is the arrival rate per unit time-unit space, and $\alpha$ and $\beta$ are probability distributions. The arrival time $t$ of an eddy, its location $z$ in space, its amplitude $a$ as well as its scale $b$ are all random and governed by $N$. By the superposition of these eddies appropriately decaying in time, a stationary velocity field $u$ is constructed as

$$
u(x, t)=\int_{(-\infty, t] \times Q} N(d s, d z, d a, d b) \exp (-c(t-s)) a v\left(\frac{x-z}{b}\right), \quad x \varepsilon \mathbb{R}^{2}, \quad t \varepsilon \mathbb{R}
$$

where $c>0$ is the decay parameter [1]. Since the flows considered in this paper are in $\mathbb{R}^{2}$, isotropy requires the basic eddy $v$ to have a specific form. Namely, $v=\left(v_{1} ; v_{2}\right)$ corresponds to rotation around 0 with magnitude $m(r)$ at distance $r$ from 0 , where $m: \mathbb{R} \rightarrow \mathbb{R}_{+}$is continuous and has support $[0 ; 1]$. The specific equations for $v$ are

$$
v_{1}(\mathbf{x})=-\frac{x_{2}}{r} m(r), \quad v_{2}(\mathbf{x})=\frac{x_{1}}{r} m(r)
$$

where $x=\left(x_{1} ; x_{2}\right)$ and $r=|x| \in[0 ; 1]$. As a rotation on $\mathbb{R}^{2}, v$ is incompressible, that is, divergence free. We let it vanish outside the unit disk through the choice of $m$. Then, every eddy is a rotation, since it is translation, amplification and dilation of $v$. As a superposition of these eddies, the velocity field $u$ is both incompressible and isotropic.

Caglar [8] has generalized the Cinlar velocity field is dependence of the decay parameter on the spatial variable $x$ and the index $q$ as

$$
c_{q}(x)=c\left|\frac{x-z}{b}\right|^{2 \gamma}, \quad q=(z, a, b)
$$

for each $x \in \mathbb{R}^{d}$ where $c>0$ as before and $\gamma>0$. For $x \in \mathbb{R}^{2}, t \in \mathbb{R}$; the velocity field is given by

$$
u(x, t)=\int_{-\infty}^{t} \int_{Q} N(d s, d z, d a, d b) \exp \left(-c|(x-z) / b|^{2 \gamma}(t-s)\right) a v\left(\frac{x-z}{b}\right),
$$


The correlation of velocity field is computed as [8],

$$
R_{i j}(\mathbf{x}, t)=\lambda \int_{\mathbb{R}^{2}} d z \int_{\mathbb{R}} \alpha(d a) a^{2} \int_{\mathbb{R}+} \beta(d b) \frac{a^{2} b^{2} \exp \left(-c|z|^{2 \gamma}|t|\right)}{c|z|^{2 \gamma}+c\left|z+\frac{x}{b}\right|^{2 \gamma}} v_{i}(z) v_{j}\left(z+\frac{x}{b}\right)
$$

We choose the distribution $\beta$ of $b$ as Gamma distribution given by,

$$
\beta(d b)=\frac{b^{\theta-1} \exp (-b / \eta)}{\Gamma(\theta) \eta^{\theta}}, \quad b>0, \quad \theta, \eta>0
$$

Hence, we have

$$
R_{i j}(\mathbf{x}, t)=\frac{\lambda}{c} \int_{\mathbb{R}} \alpha(d a) a^{2} \int_{\mathbb{R}^{2}} d z \exp \left(-c|z|^{2 \gamma}|t|\right) \int_{\mathbb{R}+} d b \frac{b^{\theta-1} \exp (-b / \eta)}{\Gamma(\theta) \eta^{\theta}} \frac{v_{i}(z) v_{j}\left(z+\frac{x}{b}\right)}{|z|^{2 \gamma}+\left|z+\frac{x}{b}\right|^{2 \gamma}}
$$

for $x \in \mathbb{R}^{2}$ and $t \in \mathbb{R}^{2}$.

\section{THE ENERGY SPECTRUM}

The Fourier transform $E$ of $R$ is called the spectral density tensor given by

$$
E_{i j}(k, w)=\frac{1}{(2 \pi)^{2}} \int_{\mathbb{R}} \int_{\mathbb{R}^{2}} \exp (-\mathbf{i}(k \cdot x+w t)) R_{i j}(x, t) d x d t
$$

The energy spectrum $\varepsilon$ is obtained from $E$ for Cinlar velocity fields [8]

$$
\varepsilon(|k|)=\frac{\pi^{3}|k|}{c} \int_{\mathbb{R}} \alpha(d a) a^{2} \int_{\mathbb{R}+} \beta(d b) b^{4} \widehat{v}(b k) \widehat{v}(-b k)
$$

where $\widehat{v}(k)=\frac{1}{(2 \pi)^{2}} \int_{\mathbb{R}^{2}} \exp (-\mathbf{i} k \cdot x) v(x) d x$. Turbulent energy per unit mass is defined in physical space and is equivalent to

$$
\frac{1}{2} \int_{\mathbb{R}^{2}}\left(E_{11}+E_{22}\right) d k=\frac{1}{2} \int_{\mathbb{R}^{2}} \sum_{j=1}^{2} \frac{1}{4 \pi^{2}} \int_{\mathbb{R}^{2}} \exp (-\mathbf{i} k \cdot x) R_{j j}(x, 0) d x d k=: \int_{0}^{\infty} \varepsilon(|k|) d|k|
$$

as shown in [8]. From (12), we want to show form of turbulent energy's the spectrum is

$$
\int_{\mathbb{R}^{2}} \exp (-\mathbf{i} k \cdot x) R_{j j}(x, 0) d x=\frac{\lambda \mathbb{E}\left(a^{2}\right)}{c \Gamma(\theta)} \int_{\mathbb{R}^{2}} d z v_{j}(z) \int_{\mathbb{R}+} d b b^{\theta-1} \exp (-b) \int_{\mathbb{R}^{2}} d x \frac{\exp (-\mathbf{i} b k \cdot x) v_{j}\left(z+\frac{x}{b \eta}\right)}{|z|^{2 \gamma}+\left|z+\frac{x}{b \eta}\right|^{2 \gamma}}
$$

where the distribution is gamma distribution. By rearranging the integrals and making a change of variable $z+\frac{x}{b \eta}=x^{\prime}$, we get

$$
\begin{aligned}
\int_{\mathbb{R}^{2}} \exp (-\mathbf{i} k \cdot x) R_{j j}(x, 0) d x= & \frac{\lambda \mathbb{E}\left(a^{2}\right) \eta^{2}}{c \Gamma(\theta)} \int_{\mathbb{R}^{2}} d z \exp (\mathbf{i} b \eta k \cdot z) v_{j}(z) \int_{\mathbb{R}+} d b b^{\theta+1} \exp (-b) \\
& \cdot \int_{\mathbb{R}^{2}} d x \frac{\exp (-\mathbf{i} b \eta k \cdot x) v_{j}(x)}{|z|^{2 \gamma}+|x|^{2 \gamma}} .
\end{aligned}
$$

We get the energy spectrum as

$$
\begin{aligned}
\varepsilon(b \eta|k|)= & \frac{\lambda \mathbb{E}\left(a^{2}\right) \eta^{2}}{8 \pi^{2} c \Gamma(\theta)} \int_{\mathbb{R}+} d b b^{\theta+1} \exp (-b) \int_{\mathbb{R}^{2}} d z \exp (\mathbf{i} b \eta k \cdot z) \\
& \cdot \int_{\mathbb{R}^{2}} d x \frac{\exp (-\mathbf{i} b \eta k \cdot x)\left(v_{1}(x) v_{1}(z)+v_{2}(x) v_{2}(z)\right)}{|z|^{2 \gamma}+|x|^{2 \gamma}}
\end{aligned}
$$


From radial transform as follows

$$
\begin{aligned}
& k=\left(k_{1}, k_{2}\right)=(|k| \cos \alpha,|k| \sin \alpha) \\
& x=\left(x_{1}, x_{2}\right)=(|x| \cos \varphi,|x| \sin \varphi) \\
& z=\left(z_{1}, z_{2}\right)=(|z| \cos \psi,|z| \sin \psi)
\end{aligned}
$$

we get

$$
\begin{aligned}
\varepsilon(b \eta|k|)= & \sum_{j=1}^{2} \frac{\lambda \mathbb{E}\left(a^{2}\right) \eta^{2}}{8 \pi^{2} c \Gamma(\theta)} \int_{\mathbb{R}+} d b \int_{0}^{2 \pi} d \psi \int_{0}^{1} d|z| \int_{0}^{2 \pi} d \varphi \int_{0}^{1} d|x| \exp (\mathbf{i} b|k||z| \cos (\psi-\alpha))|z| v_{j}(|z|, \psi) \\
& \cdot \exp (-\mathbf{i} b|k||x| \cos (\varphi-\alpha)) \frac{v_{j}(|x|, \varphi)}{|x|^{2 \gamma}+|z|^{2 \gamma}}
\end{aligned}
$$

We use properties of the Bessel functions

$$
\begin{aligned}
\exp (\mathbf{i} z \cos (\theta)) & =\sum_{n=-\infty}^{\infty} \mathbf{i}^{n} J_{n}(z) \exp (\mathbf{i} n \theta) \\
& =\sum_{n=0}^{\infty} J_{n}(z)\left[\mathbf{i}^{n} \exp (\mathbf{i} n \theta)+(-1)^{n} \mathbf{i}^{-n} \exp (-\mathbf{i} n \theta)\right]
\end{aligned}
$$

since $J_{-n}(x)=(-1)^{n} J_{n}(x)$. Using (4) and substituting (15), we get

$$
\begin{aligned}
\varepsilon(b \eta|k|)= & \frac{\lambda \mathbb{E}\left(a^{2}\right) \eta^{2}}{8 \pi^{2} c \Gamma(\theta)} \int_{\mathbb{R}+} e^{-b} b^{\theta+1} d b \int_{0}^{1} d|z| \int_{0}^{2 \pi} d \psi \int_{0}^{1} d|x| \int_{0}^{2 \pi} d \varphi m(|z|) m(|x|)|z||x| \frac{\cos (\psi-\varphi)}{|x|^{2 \gamma}+|z|^{2 \gamma}} \\
& \sum_{n=0}^{\infty} J_{n}(\eta b|k||z|)\left[\mathbf{i}^{n} \exp (\mathbf{i} n(\psi-\alpha))+(-1)^{n} \mathbf{i}^{-n} \exp (-\mathbf{i} n(\psi-\alpha))\right] \\
& \cdot \sum_{m=0}^{\infty} J_{m}(\eta b|k||x|)\left[^{m} \exp (\mathbf{i} m(\pi-(\varphi-\alpha)))+(-1)^{m} \mathbf{i}^{-m} \exp (-\mathbf{i} m(\pi-(\varphi-\alpha)))\right]
\end{aligned}
$$

Making some algebraic computations and using trigonometric properties, the above expression is simplified due to orthogonality of sin and cos functions and we get only the term corresponding to $m=1, n=1$ as

$$
\varepsilon(b \eta|k|)=\frac{4 \pi^{2} \lambda \mathbb{E}\left(a^{2}\right) \eta^{2}}{c \Gamma(\theta)} \int_{\mathbb{R}+} e^{-b} b^{\theta+1} d b \int_{0}^{1} m(|z|)|z| J_{1}(\eta b|k||z|) d|z| \int_{0}^{1} m(|x|)|x| \frac{J_{1}(\eta b|k||x|)}{|x|^{2 \gamma}+|z|^{2 \gamma}} d|x|
$$

Asymptotic expansion of Bessel functions as $|k| \rightarrow \infty[6]$

$$
J_{1}(k r) \propto \frac{1}{2}\left(\frac{2}{\pi k r}\right)^{1 / 2}\left[(-\mathbf{i})^{1+1 / 2} \exp (\mathbf{i} k r)+\mathbf{i}^{1+1 / 2} \exp (-\mathbf{i} k r)\right] .
$$

Firstly, using above property we compute product of Bessel functions as

$$
\begin{aligned}
J_{1}(\eta b|k||z|) J_{1}(\eta b|k||x|) & =\frac{1}{2 \pi \eta b|k| \sqrt{|x||z|}}\left[(-\mathbf{i})^{3 / 2} \exp (\mathbf{i} \eta b|k||z|)+\mathbf{i}^{3 / 2} \exp (-\mathbf{i} \eta b|k||z|)\right] \\
& \cdot\left[(-\mathbf{i})^{3 / 2} \exp (\mathbf{i} \eta b|k||x|)+\mathbf{i}^{3 / 2} \exp (-\mathbf{i} \eta b|k||x|)\right] \\
& =\frac{1}{2 \pi \eta b|k| \sqrt{|x||z|}}\left[-\mathbf{i}^{3} \exp (\mathbf{i} \eta b|k|(|x|+|z|))+-\mathbf{i}^{3 / 2} \mathbf{i}^{3 / 2} \exp (\mathbf{i} \eta b|k|(|x|-|z|))\right. \\
& \left.+\mathbf{i}^{3 / 2}-\mathbf{i}^{3 / 2} \exp (-\mathbf{i} \eta b|k|(|x|-|z|))+\mathbf{i}^{3} \exp (-\mathbf{i} \eta b|k|(|x|+|z|))\right] \\
& =\frac{1}{2 \pi \eta b|k| \sqrt{|x||z|}}[\mathbf{i} \cos (\eta b|k|(|x|+|z|))-\sin (\eta b|k|(|x|+|z|))-\mathbf{i} \cos (\eta b|k|(|x|+|z|)) \\
& -\sin (\eta b|k|(|x|+|z|))-\cos (\eta b|k|(|x|-|z|))-\mathbf{i} \sin (\eta b|k|(|x|-|z|)) \\
& -\cos (\eta b|k|(|x|-|z|))+\mathbf{i} \sin (\eta b|k|(|x|-|z|))] \\
& =-\frac{1}{\pi \eta b|k| \sqrt{|x||z|}}(\sin (\eta b|k|(|x|+|z|))+\cos (\eta b|k|(|x|-|z|)))
\end{aligned}
$$


After above computations, we get

$$
\begin{aligned}
& \varepsilon(b \eta|k|) \propto \frac{-4 \pi \lambda \mathbb{E}\left(a^{2}\right) \eta}{c|k| \Gamma(\theta)} \int_{\mathbb{R}+} e^{-b} b^{\theta} d b \int_{0}^{1} \int_{0}^{1} m(|z|) m(|x|) \frac{(|x||z|)^{1 / 2}}{|x|^{2 \gamma}+|z|^{2 \gamma}} \\
& \cdot \quad[\sin (\eta b|k|(|x|+|z|))+\cos (\eta b|k|(|x|-|z|))] d|z| d|x| .
\end{aligned}
$$

Making change of variable as $\eta b|k|=b^{\prime}$,

$$
\begin{aligned}
\varepsilon(|k|) \propto & \frac{-4 \pi \lambda \mathbb{E}\left(a^{2}\right)}{c \eta^{\theta} \Gamma(\theta)|k|^{\theta+2}} \int_{\mathbb{R}+} e^{-b /|k| \eta} b^{\theta} d b \\
& \cdot \quad \int_{0}^{1} \int_{0}^{1} m(|z|) m(|x|) \frac{(|x||z|)^{1 / 2}}{|x|^{2 \gamma}+|z|^{2 \gamma}}(\sin (b(|x|+|z|))+\cos (b(|x|-|z|))) d|z| d|x|
\end{aligned}
$$

and new change of variables as $b|x|=|x|^{\prime}$ and $b|y|=|y|^{\prime}$, we get

$$
\begin{aligned}
\varepsilon(|k|) \propto & \frac{-4 \pi \lambda \mathbb{E}\left(a^{2}\right)}{c \eta^{\theta} \Gamma(\theta)|k|^{\theta+2}} \int_{\mathbb{R}+} e^{-b /|k| \eta} b^{\theta} d b \\
& \cdot \int_{0}^{1 / b} \int_{0}^{1 / b} m\left(\frac{|z|}{b}\right) m\left(\frac{|x|}{b}\right) \frac{(|x||z|)^{1 / 2}}{|x|^{2 \gamma}+|z|^{2 \gamma}} b^{2 \gamma-3}(\sin (|x|+|z|)+\cos (|x|-|z|)) d|z| d|x|
\end{aligned}
$$

After rearrangement,

$$
\varepsilon(|k|) \propto \frac{4 \pi \lambda \mathbb{E}\left(a^{2}\right)|k|^{-\theta-2}}{c \eta^{\theta} \Gamma(\theta)} \int_{\mathbb{R}+} e^{-b /|k| \eta} b^{\theta} f(b) d b
$$

where

$$
f(b)=\int_{0}^{1 / b} \int_{0}^{1 / b} m\left(\frac{|z|}{b}\right) m\left(\frac{|x|}{b}\right) \frac{(|x||z|)^{1 / 2}}{|x|^{2 \gamma}+|z|^{2 \gamma}} b^{2 \gamma-3}(\sin (|x|+|z|)+\cos (|x|-|z|)) d|z| d|x|
$$

\section{DISCUSSION}

In this study, the effect of gamma distribution on computation of the energy spectrum has investigated. As a conclusion when a gamma distribution is used as a eddy radial distribution, we obtain a gamma like functions of $|k|$ in addition to power of $|k|$. Lundgren [6] has showed that in strained spiral vortex model, the energy spectrum for small scale structure is found as $\varepsilon(|k|)=A k^{-5 / 3} e^{\left[-\frac{2}{3}\left(v k^{2} / a\right)\right]}$. As future work, we aim to seek a distribution that yields an exponential term in the energy spectrum for Cinlar velocity field as in spiral vortex. Furthermore, the velocity field will be used for modeling the effect of small scales in large eddy simulation.

\section{REFERENCES}

1. E. Cinlar, On a Random Velocity Field, Princeton University, 1993.

2. E. Cinlar, Isotropic Velocity Fields of Poisson Shot Noise Type, Princeton University, 1994.

3. M. Caglar, Simulation of homogeneous and incompressible Cinlar flows, Appl. Math. Modelling, 24, pp. 297-314 (2000).

4. M. Caglar, Dispersion of mass by two-dimensional homogeneous and incompressible Cinlar flows, Appl. Math. Modelling, 27, pp. 997-1011 (2003).

5. M. Caglar, and E. Cinlar, Lyapunov Exponents of Poisson Shot-Noise Velocity Fields, Stochastic Processes and their Applications, 94, pp. 29-49 (2001).

6. T. S. Lundgren, Strained spiral vortex model for turbulent fine structure, Phys. Fluids, 25-12, pp. 2195-2203 (1982).

7. M. Caglar, T. Bilal, L. Piterbarg, Lagrangian Prediction and Correlation Analysis with Eulerian Data, Turkish J. Earth Sciences, 20, pp. 343-358 (2011).

8. M. Caglar, Velocity Fields with Power-Law Spectra for Modeling Turbulent Flows, Applied Mathematical Modelling, 31, pp. 1934-1946 (2007). 